\title{
Autologous Serum Eye Drops versus Artificial Tear Drops for Dry Eye Disease: A Systematic Review and Meta-Analysis of Randomized Controlled Trials
}

\author{
Leying Wang $^{\mathrm{a}}$ Kai Cao ${ }^{\mathrm{a}}$ Zhenyu Wei $^{\mathrm{a}}$ Christophe Baudouin $^{\mathrm{a}-\mathrm{c}}$ \\ Antoine Labbéa-c Qingfeng Liang ${ }^{\text {a }}$ \\ aBeijing Institute of Ophthalmology, Beijing Tongren Eye Center, Beijing Tongren Hospital, Capital Medical \\ University, Beijing Key Laboratory of Ophthalmology and Visual Sciences, Beijing, China; ${ }^{b}$ IHU FOReSIGHT, Paris and \\ Versailles Saint-Quentin-en-Yvelines University, Versailles, France; ' Sorbonne Université, INSERM, CNRS, Institut de \\ la Vision, Paris, France
}

\section{Keywords}

Dry eye disease $\cdot$ Autologous serum eye drops · Artificial tear drops · Randomized controlled trials $\cdot$ Meta-analysis

\begin{abstract}
Objective: To compare the efficacy of autologous serum (AS) eye drops and artificial tears (AT) in dry eye disease (DED). Methods: Five databases (PubMed, Science Direct, the Cochrane Library, the Chinese National Knowledge Infrastructure, and the Wanfang Database) were searched for randomized controlled trials (RCTs). Efficacy was evaluated in terms of the Ocular Surface Disease Index (OSDI), Schirmer I test, tear break-up time (TBUT), and fluorescein and rose bengal staining of ocular surface. The estimated effects of AS or AT were expressed as a proportion with the $95 \%$ confidence interval and plotted on a forest plot. Results: Seven RCTs with 267 subjects were included in the meta-analysis. For most of the studies, subjects' age was around 50 years old, and the mostly treatment duration was within 8 weeks. The followup results showed that the OSDI after AS treatment was lower than that after the AT treatment: the mean difference (MD) was $-10.75(95 \% \mathrm{Cl},-18.12$; -3.39$)$ points. There was no difference on the Schirmer I test after treatment between the two groups: the MD was $1.68(95 \% \mathrm{Cl},-0.65 ; 4.00) \mathrm{mm}$. The
\end{abstract}

TBUT of the AS group was longer than that of the AT group, with an MD of $4.53(95 \% \mathrm{Cl}, 2.02 ; 7.05)$ s. There was no statistically significant difference on fluorescein staining score of the ocular surface between the AS group and the AT group, the MD was $-2.53(95 \% \mathrm{Cl},-6.08 ; 1.03)$ points. The rose bengal staining score of the AS group was slightly lower than that of the AT group after treatment: the MD was -0.78 ( $95 \%$ $\mathrm{Cl},-1.34 ;-0.22)$ points. Conclusion: AS could be an effective treatment for DED, improving OSDI, TBUT, and rose bengal staining score. Further RCTs with large samples and longterm follow-up are still needed to determine the exact role of AS in the management of DED.

ㄷ) 2019 S. Karger AG, Basel

\section{Introduction}

Dry eye disease (DED) is a multifactorial disease of ocular surface with tear film instability, hyperosmolarity, ocular surface inflammation, and neurosensory abnormalities [1]. Population-based studies have reported that the prevalence rate of DED was 5-34\% worldwide [2] and 1.5-2.2 times higher in Asians than in Caucasians [3]. In 2010 , the number of DED cases diagnosed with symptoms reached 394.13 million in China [4]. Additionally,

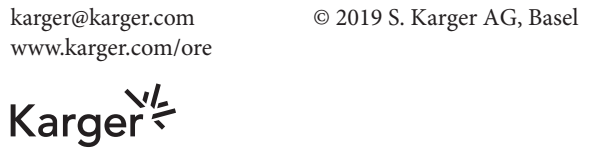

Prof. Qingfeng Liang, Beijing Institute of Ophthalmology Beijing Tongren Eye Center, Beijing Tongren Hospital Capital Medical University, Beijing Key Laboratory of Ophthalmology and Visual Sciences, Beijing 100005 (China) lqflucky@163.com 
with the popularity of video screens, environmental pollution, and the aging of the population, DED has become a major public health problem. It brings great economic burden by the rise of medical expenses and the decline of social productivity. DED always results in chronic pain and negative daily activities and work, and influences their life quality $[4,5]$.

The therapy and management subcommittee of the 2017 Dry Eye Workshop II (DEWS II) proposed that graded treatment could be administered to DED patients, and if the initial and second-level treatments failed, thirdlevel treatment, such as autologous serum (AS), should be considered [6]. AS is centrifuged from blood, without fibrinogens, and is similar to human tears in biomechanical and biochemical properties, for example, hydrogen ion concentration $(\mathrm{pH})$, osmolarity, and components [7]. Several beneficial mechanisms of AS in treating DED have been studied, including the nourishment of nerve and epithelium and the promotion effect on the proliferation and migration of corneal epithelium [7]. Meanwhile, AS can increase the density of goblet cells and the expression of mucin in conjunctival tissue [8]. More importantly, AS inhibits the release of inflammatory cytokines, which might alleviate the vicious cycle between ocular surface inflammation and DED, and ultimately maintains the homeostasis of the microenvironment of the ocular surface [9]. In contrast to artificial tears (AT), to date only rare toxic or allergic reactions have been reported using AS in DED [10-12].

Since Ralph et al. [13] first proposed the use of AS for $\mathrm{DED}$, a large number of studies have been conducted to evaluate its efficacy in severe or refractory DED. However, data on the effect of AS for DED remains equivocal [14, 15]. To provide sufficient and powerful evidence to assess the effectiveness of AS for DED, a systematic review was conducted with a meta-analysis (randomized controlled trials, RCTs) focused on the treatment of DED with AS.

\section{Methods}

\section{Inclusion and Exclusion Criteria}

All studies included in this review were RCTs. The subjects were DED patients, regardless of the participants' age or sex or etiology of disease. The intervention measurement should be AS therapy, and the control group should be given AT treatment, with or without combination of other therapy. The evaluation indicators must contain at least one of Ocular Surface Disease Index (OSDI), Schirmer I test, tear break-up time (TBUT), fluorescein staining, and rose bengal staining.

Studies would be excluded if the study design was not RCT or subjects or intervention measurement did not meet the inclusion criteria. Besides, if the original quantitative data of effectiveness of AS or AT was not extractable or the appraisal system could not be combined with other studies, the study would be excluded.

\section{Outcome Measurement}

Outcome evaluation included the analysis about subjective symptoms, Schirmer I test, TBUT, fluorescein staining, and rose bengal staining. Subjective symptoms were measured by OSDI, which is the most extensively used questionnaire for DED. Schirmer I test was performed using a strip placed in the lower conjunctival sac for 5 min without anesthesia. TBUT was the average of two or three measurements. For fluorescein staining, we analyzed studies which used same graded system. Briefly, the cornea was horizontal divided into three equal compartments, and each zone was graded from 0 to 3 . For rose bengal staining, we analyzed studies using rose bengal $1 \%$ and a scale of 9 points.

\section{Databases and Search Strategy}

The following databases were analyzed: PubMed, Science Direct, the Cochrane Library, the Chinese National Knowledge Infrastructure, and the Wanfang Database, with one or a combination of the following terms: autologous serum, artificial tears, comparison, random, and randomized. Details of the search strategy are available in online supplementary Appendix 1 (see www.karger.com/doi/10.1159/000505630). The publication date was from the creation of the databases to December 31, 2018.

\section{Data Extraction}

We extracted data of outcomes measured at the last follow-up time. In this meta-analysis, all extracted data, including OSDI, TBUT, Schirmer I test, fluorescein staining score, and rose bengal staining score, were continuous data and we extracted the mean value and standard deviation (SD).

\section{Assessment on Risk of Bias and Article Quality}

We used the risk of bias tool recommended by the Cochrane Collaboration [16] to access the risk of bias on the following six aspects: random sequence generation, allocation concealment, blinding of participants and personnel, blinding of outcome assessors, incomplete outcome data, and selective outcome reporting. Specifically, we carefully reviewed the studies included and checked whether there was a description of how the random sequence was generated and allocated, whether and how the blinding method was applied, how the outcomes were measured, and whether there were missing data. For example, if the random sequence was generated using a random number table or pseudo-random numbers using statistical software, the risk of bias was assessed as "low" level; otherwise, if there was evidence showing that the random sequence was generated in an inappropriate way, the risk of bias on random sequence generation was assessed as a "high" level. If no information could be obtained or it was difficult to make a judgment, the risk of bias was assessed as "unclear."

\section{Data Synthesis and Statistical Analysis}

In this study, the mean value of the AS group minus the mean value of the AT group (mean difference, MD) with the $95 \%$ confidence interval (CI) was used to estimate continuous outcomes. We defined resolution of DED as the increase of Schirmer I, extension of TBUT, the decrease of OSDI, fluorescein staining score, and rose bengal score. All the analyses were conducted with the opensource R program (version 3.4.4). Before estimating the pooled 
Fig. 1. Flowchart of selection of original pa-

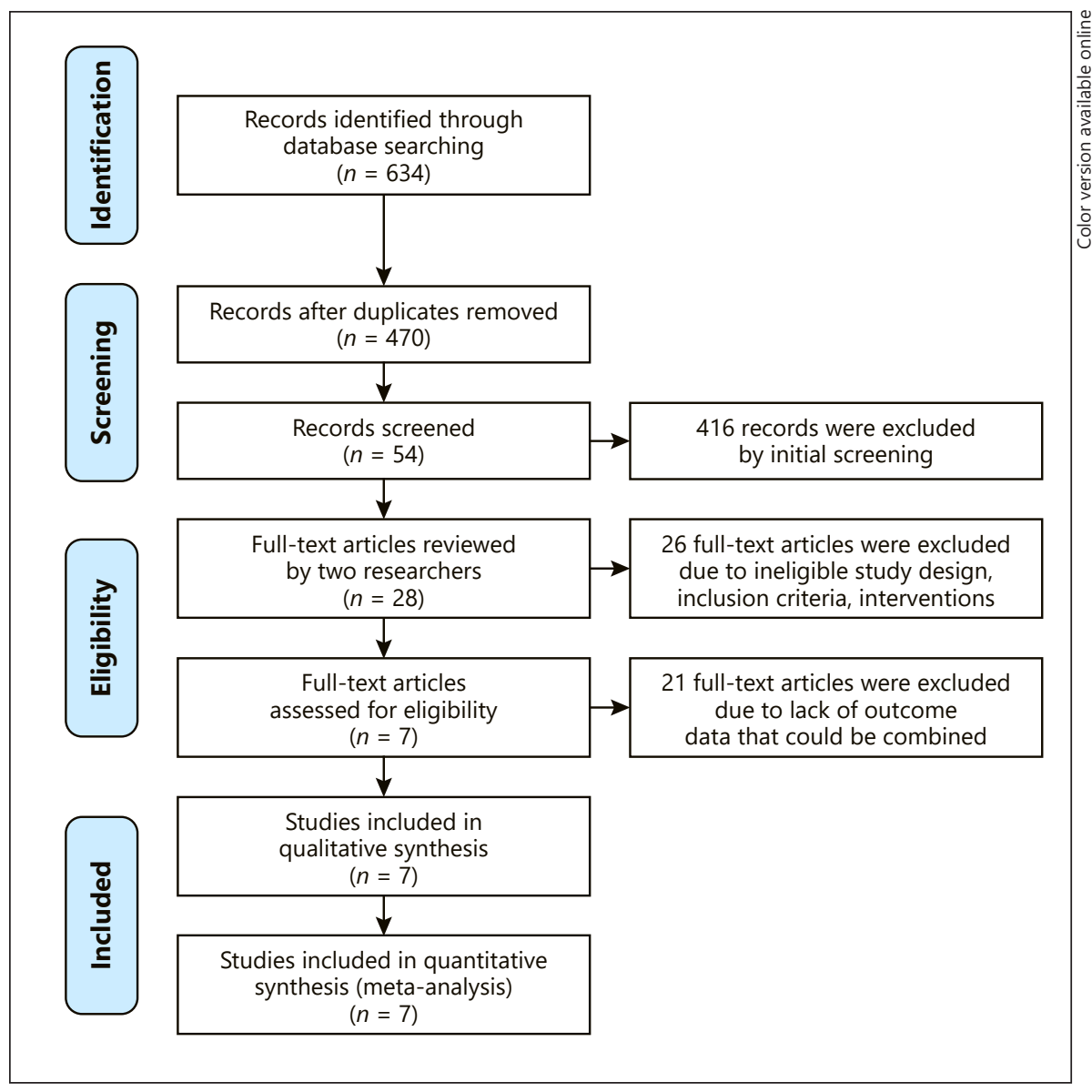
pers.

Table 1. Characteristics of the RCTs included in the meta-analysis

\begin{tabular}{|c|c|c|c|c|c|c|c|c|}
\hline \multirow[t]{2}{*}{ First author } & \multirow{2}{*}{$\begin{array}{l}\text { Publication } \\
\text { year }\end{array}$} & \multirow{2}{*}{$\begin{array}{l}\text { Study } \\
\text { design }\end{array}$} & \multicolumn{2}{|c|}{ Sample size } & \multicolumn{2}{|c|}{ Subjects' age, years } & \multirow{2}{*}{$\begin{array}{l}\text { Concentration } \\
\text { (frequency) }\end{array}$} & \multirow{2}{*}{$\begin{array}{l}\text { Treatment } \\
\text { duration }\end{array}$} \\
\hline & & & AS & $\mathrm{AT}$ & AS & $\mathrm{AT}$ & & \\
\hline Kojima & 2005 & RCT & 10 & 10 & $65.4 \pm 9.7$ & $62.3 \pm 12.5$ & $20 \%$ (6 times/day) & 2 weeks \\
\hline Noda-Tsuruya & 2006 & RCT & 12 & 15 & $28.8 \pm 5.3$ & $31.2 \pm 6.2$ & $20 \%$ (6 times/day) & 6 months \\
\hline Urzua & 2012 & crossover & 6 & 6 & \multicolumn{2}{|c|}{$52 \pm 6.3$} & $20 \%$ (4 times/day) & 5 weeks \\
\hline Celebi & 2014 & crossover & 20 & 20 & \multicolumn{2}{|c|}{$56.05 \pm 8.07$} & $20 \%$ (4 times/day) & 6 weeks \\
\hline Mukhopadhyay & 2015 & $\mathrm{RCT}$ & 52 & 44 & - & - & $20 \%$ (6 times/day) & 6 weeks \\
\hline Semeraro & 2016 & RCT & 12 & 12 & $54.67 \pm 15.41$ & $54.0 \pm 7.27$ & $50 \%$ (5 times/day) & 1 year \\
\hline Yilmaz & 2017 & crossover & 24 & 24 & \multicolumn{2}{|c|}{$25 \pm 4.02$} & $40 \%$ & 2 months \\
\hline
\end{tabular}

effect, the heterogeneity across studies was first assessed using the $\mathrm{Q}$ test and $I^{2}$ statistic. $I^{2}$ describes the percentage of variability in effect estimates that is due to heterogeneity rather than to chance. If $I^{2}$ was below $50 \%$, a small heterogeneity in the study was considered and a fixed-effect model was used to pool the effect size; otherwise a random-effects model was applied and a sensitivity analysis was done [17]. The publication bias was evaluated with Egger's test and the significance level was set at 0.05, two-tailed [18].

\section{Results}

Seven studies with 267 subjects were included (Table 1). The paper selection process is shown in Figure 1. Specifically, we identified 634 records by database searching, with 470 left after duplications removed. 416 records were removed further after initial screening, and another 


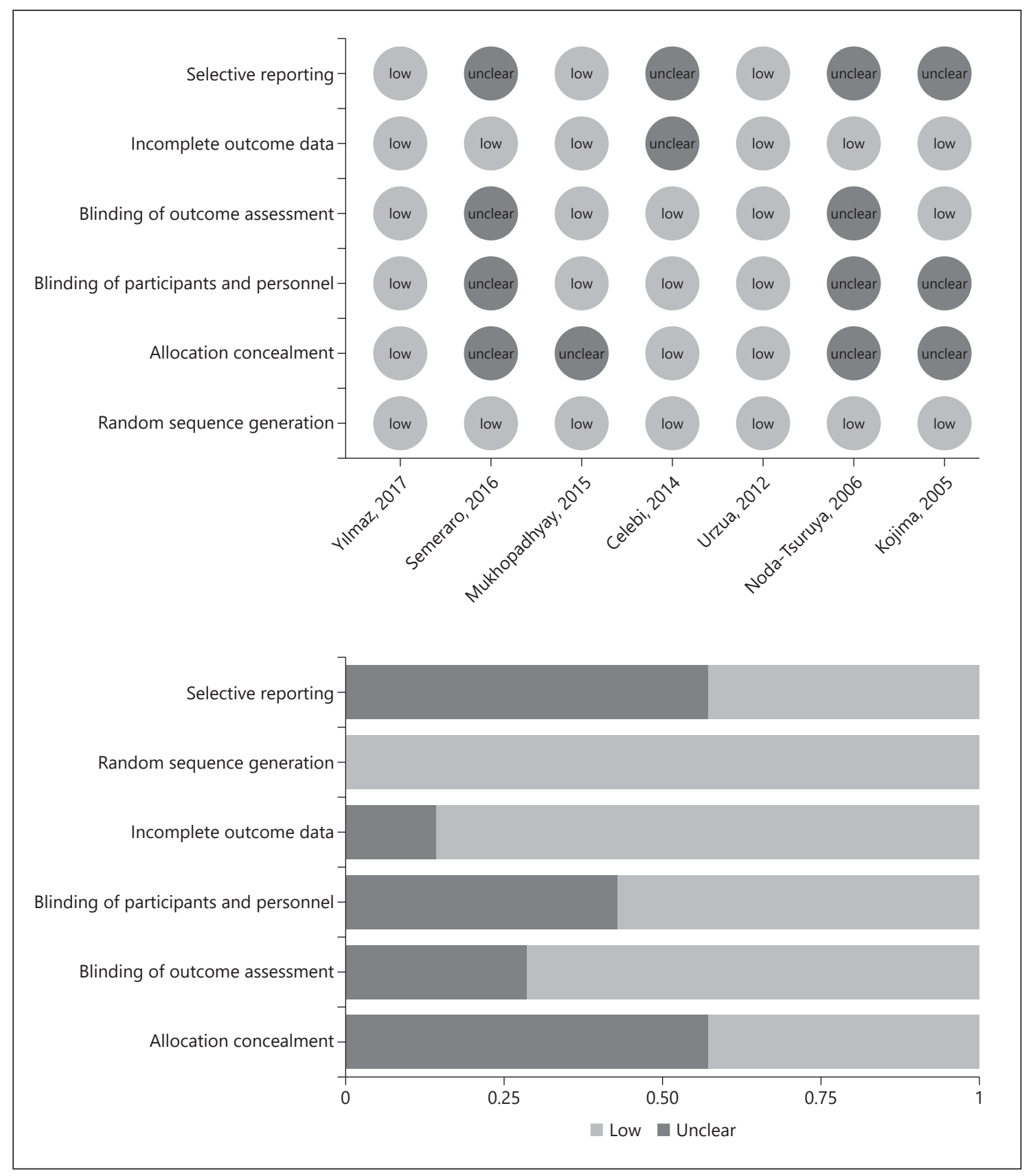

Fig. 2. Risk of bias analysis of seven DED studies with AS treatment.

26 full-text articles were excluded because they were not of the RCT design or their subjects were not meeting the inclusion criteria or the intervention measurement was not AS and AT. Besides, another 21 full-text articles were excluded due to lack of extractable outcome data that could be combined. Seven studies were included at last.

The characteristics of the included studies is shown in Table 1. Three RCTs used a crossover design. The pa- tients were all adults with a wide age range, and most were around 50 years old or above. The concentration of serum was $20 \%, 40 \%$, and $50 \%$, used by 100,24 , and 12 subjects, respectively. The frequency of eye drops ranged from four to six times a day, and the treatment duration ranged from 2 weeks to 1 year but mostly was within 8 weeks.

Analysis on risk of bias (Fig. 2) showed that all studies generated the random sequence appropriately; thus, there 


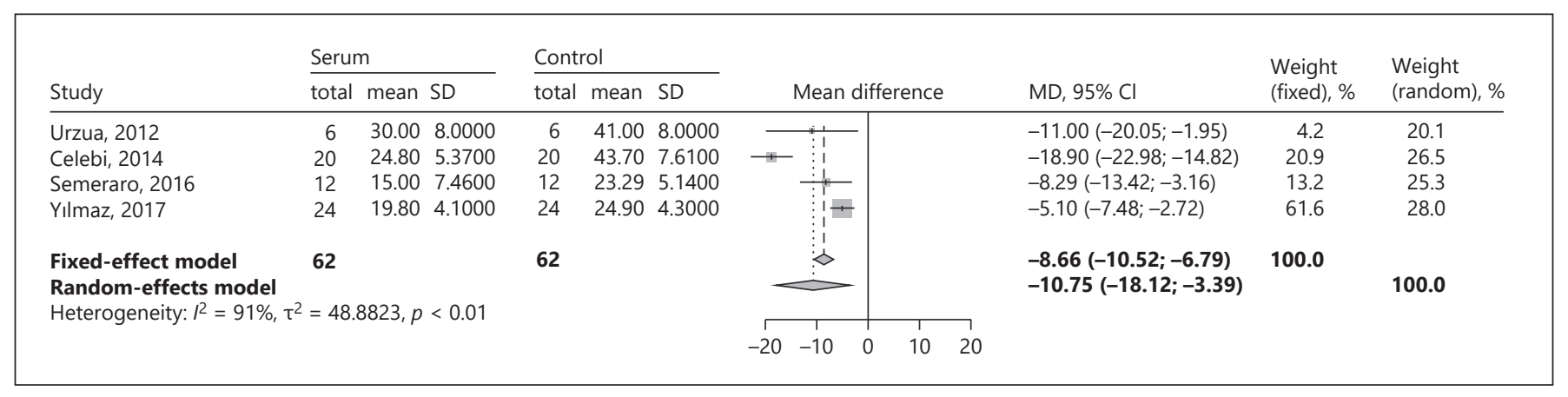

Fig. 3. Forest plot of Ocular Surface Disease Index after AS or AT treatment.

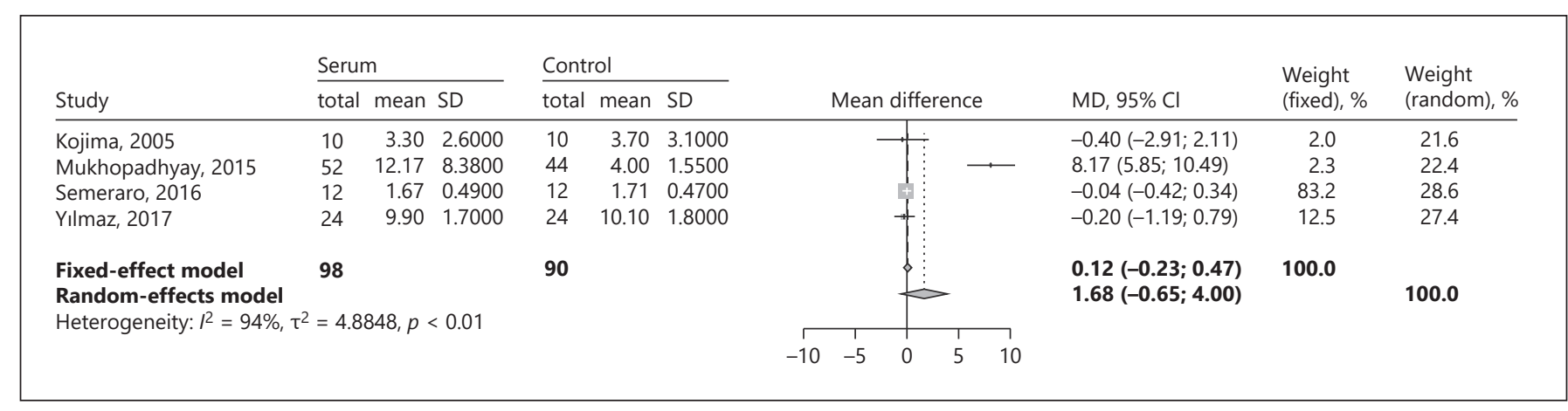

Fig. 4. Forest plot of Schirmer I test after AS or AT treatment.

was a low risk of bias on random sequence generation. Three studies stated clearly that the allocation was sealed with an envelope or wrapped with aluminum foil, and they had a low risk of bias on allocation concealment, while for the remaining four studies, the risk of bias was unclear. As for blinding, some studies stated clearly that patients or data evaluators or the data analyst were masked, and no evidence indicated a high risk of bias. On the outcome assessment aspect, no evidence indicated a high risk of bias. As for the incomplete data, no evidence indicated a high risk of bias either. For the selective reporting aspect, no evidence showed a high risk of bias for any study.

The OSDI of the AS group was lower than that of the AT group (Fig. 3): the MD was -10.75 (95\% CI, -18.12; $-3.39)$ points, there was a large heterogeneity across the included studies $\left(I^{2}=91 \%\right)$. We performed further sensitivity analysis, which showed that when Celebi's study was omitted, $I^{2}$ dropped to $19.6 \%$ and the conclusion remained unchanged; the MD was -8.72 (95\% CI, -10.72 ; $-6.71)$. No publication bias was found with Egger's test $(t=-0.781, p=0.516)$, as shown in Table 2. There was no

Autologous Serum Eye Drops and Dry Eye
Table 2. Egger's test for publication bias in the meta-analysis

\begin{tabular}{lrll}
\hline Variables & \multicolumn{1}{l}{$t$} & $\mathrm{df}$ & $p$ \\
\hline Ocular surface disease index & -0.781 & 2 & 0.516 \\
Schirmer I test & 0.984 & 2 & 0.429 \\
Tear break-up time & 0.089 & 3 & 0.934 \\
Fluorescein staining & -0.049 & 2 & 0.966 \\
Rose bengal staining & -0.742 & 1 & 0.594 \\
\hline
\end{tabular}

difference in the Schirmer I test between the AS group and the AT group (Fig. 4): the MD was 1.68 (95\% CI, $-0.65 ; 4.00) \mathrm{mm}$. There was a large heterogeneity across the included studies $\left(I^{2}=94 \%\right)$. We then conducted the sensitivity analysis and it turned out that when Mukhopadhyay's study was omitted, $I^{2}$ dropped to $0 \%$ and the conclusion remained unchanged: the MD was -0.07 (95\% CI, $-0.42 ; 0.29)$. No publication bias was found with the Egger test $(t=0.984, p=0.429)$.

The TBUT of the AS group was longer than that of the AT group (Fig. 5), with an MD of 4.53 (95\% CI, 2.02; 7.05) 


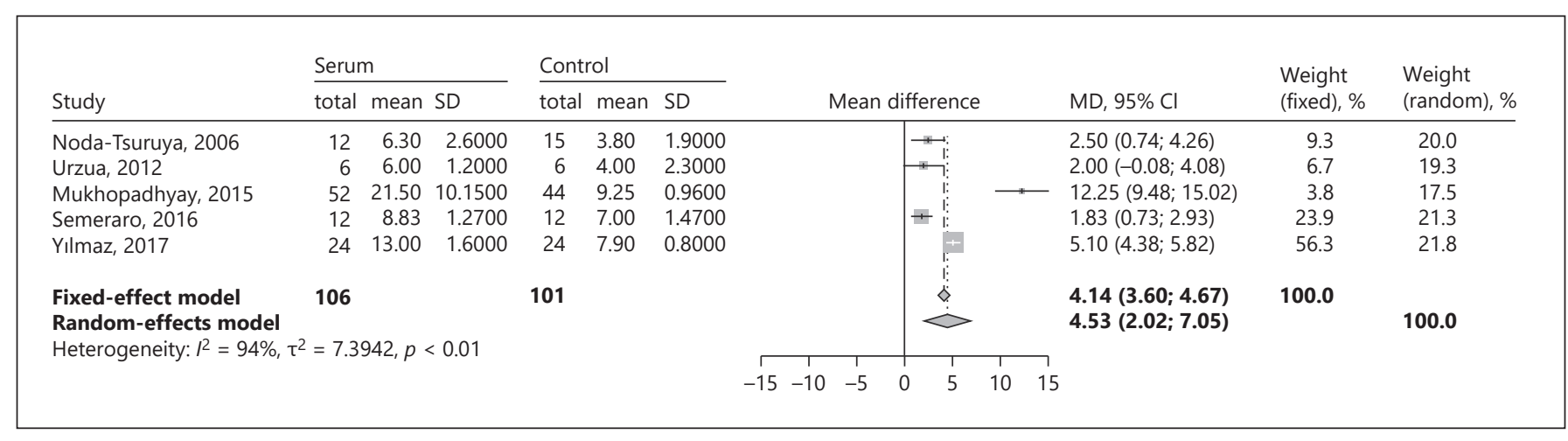

Fig. 5. Forest plot of tear break-up time after AS or AT treatment.

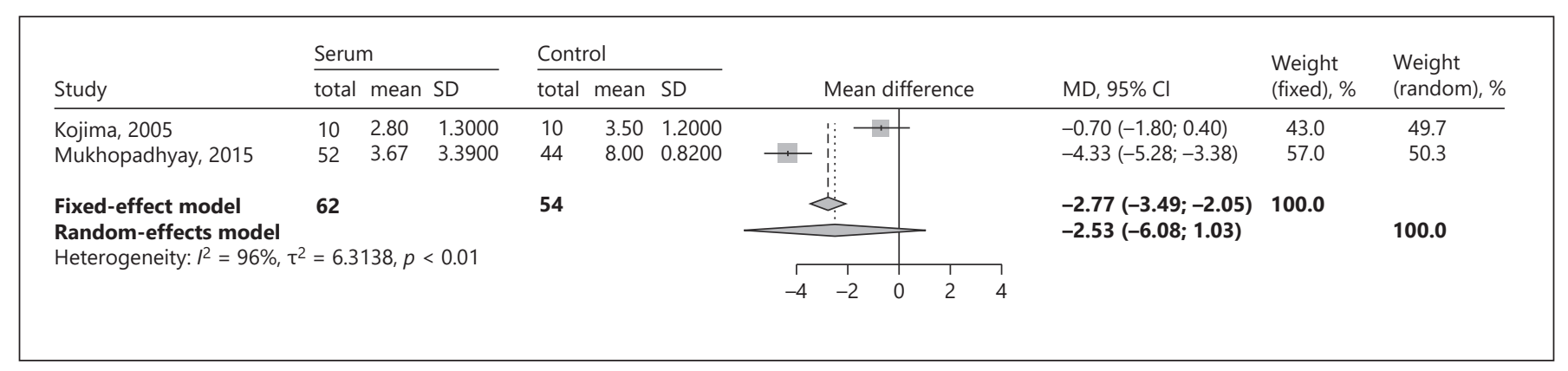

Fig. 6. Forest plot of fluorescein staining score after AS or AT treatment.

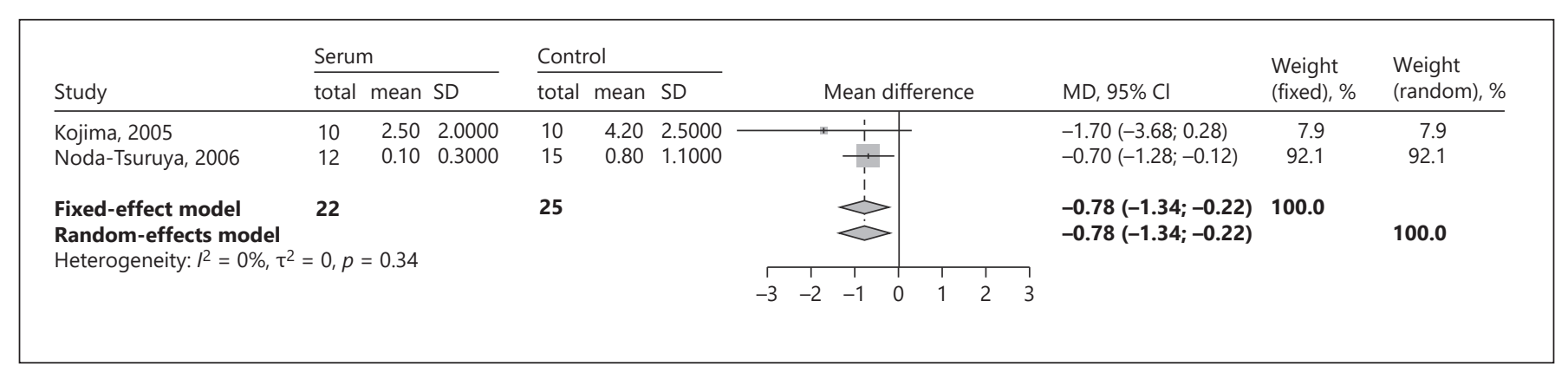

Fig. 7. Forest plot of rose bengal staining score after AS or AT treatment.

s. Substantial heterogeneity on TBUT across the studies included was found $\left(I^{2}=94 \%\right)$, because the confidence intervals of the different studies did not overlap well. No single study showed a shorter TBUT in the AT group. There was no publication bias based on the Egger test $(t=0.089, p=0.934)$.

There was no statistical difference on the fluorescein staining score between the AS group and the AT group (Fig. 6); the MD was -2.53 (95\% CI, -6.08 ; 1.03) points.
There was substantial heterogeneity across the included studies $\left(I^{2}=96 \%\right)$. The rose bengal staining score of the AS group was lower than that of the AT group (Fig. 7), the MD was -0.78 ( $95 \%$ CI: $-1.34 ;-0.22)$ points. There was no heterogeneity $\left(I^{2}=0 \%\right)$ or publication bias.

For additional therapy, one study, including DED subjects after LASIK, used $0.3 \%$ hyaluronic acid, antibiotics, and low-dose steroids for 1 week postoperatively. A study of all subjects with Sjögren syndrome used 200 
mg hydroxychloroquine per day. The others only used AS or AT. Moreover, Kojima et al. included patients without punctal occlusion to evaluate the solitary effect of AS or AT. None of the included studies reported adverse effects.

\section{Discussion}

AS is used clinically for the treatment of DED, yet there is no evidence-based clinical reason to support this treatment [6]. In 2017, Pan et al. [19] published a systematic review and suggested there might be some benefit in symptoms with AS compared with AT. But they did not perform meta-analysis owing to limited quantitative data. In our study, we included seven RCTs, including the latest three RCTs, and further conducted meta-analysis. The conclusion suggested AS statistically significantly improved OSDI, TBUT, and rose bengal staining score compared to AT. However, when considering the follow-up results of Schirmer I and fluorescein staining score, no significant difference was found. Besides, no adverse effect was reported among patients treated with AS.

In this meta-analysis, all of the seven studies evaluated the subjective symptoms and showed that AS performed much better than AT in relieving symptoms. Due to the fact that ocular discomfort is the fundamental of DED, as well as the primary cause for doctor's visit, symptom remission is an important goal. We also reviewed the latest published meta-analysis that focused on DED therapy. In summary, in comparison to AT or placebo, $0.05 \%$ cyclosporine group and omega-3 group had lower OSDI $(\mathrm{MD}=4.10$ and $\mathrm{MD}=0.968)$, but carboxymethylcellulose group and sodium hyaluronate group had no statistical significance [20-23]. In consideration of the fact that AS group had significant lower OSDI $(\mathrm{MD}=10.75)$ than AT group, AS might had powerful effectiveness in comforting DED patients.

Four [24-27] of the seven studies reported Schirmer I test and all of them showed an increase in tear secretion. Nevertheless, the combined results revealed no significant difference. One of these four studies included severe DED patients, another one included moderate to severe DED subjects. Tsubota et al. [28] found that, for mild or moderate DED patients, the Schirmer I test did not have sufficiently meaningful sensitivity. Moreover, inactive AT may influence Schirmer I test results because of their direct effect on increasing tear volume. Therefore, the Schirmer I test was limited in assessing the efficacy of AS or AT in DED treatment based on the patients included.

Autologous Serum Eye Drops and Dry

Eye
All included studies demonstrated TBUT was statistically significantly longer in the AS group, except Urzua et al. [29]. TBUT is a common test to evaluate the stability of tear film, which has long been known as a hallmark of ocular health. Valim et al. [30] suggested that a clinically significant difference in TBUT was $3 \mathrm{~s}$, which was much shorter than the meta-analysis outcomes showing that the difference between AS and AT was 4.53 (95\% CI, 2.02; 7.05) s. Accordingly, we assumed that AS was superior to AT in reversing tear film stability.

Six of seven RCTs evaluated the fluorescein staining score and all showed that AS performed better than AT. Nevertheless, we combined the results of two studies, both with a total of 9 points, which suggested that there was no difference between the two groups. These two studies had substantial heterogeneity because the posttreatment result of the AS group (MD 3.67, SD 3.39) was obviously lower than the AT group result (MD 8.00, SD 0.82 ) in the study reported by Mukhopadhyay et al., which indicated that AS had better efficacy and a significant difference in CI between the studies included, which may have resulted in the negative result of the analysis.

As such, the difference in rose bengal staining between the two groups was small, while statistically significant, and probably did not have clinical significance. Firstly, rose bengal staining was nonspecific, including dead or degenerated epithelial cells, as well as those unprotected by glycocalyx or mucin [31]. Secondly, the assessment of rose bengal scores is influenced by staining time and magnification and the result can be confused with concurrent hyperemia because of the similar colors. Finally, the studies included examined patients with varying disease severity and it had been concluded that the patients with more severe ocular surface damage expressed a greater response to treatment compared to patients with relatively mild disease [30].

This review did not attempt to draw conclusions from the observation of visual acuity, corneal confocal microscopy, conjunctival impression cytology grading, and tear lysozyme or lactoferrin levels, because these outcomes were quantitatively reported in fewer than two studies each. Even though the meta-analysis provided a synthetically and objective evaluation of the efficacy of AS in the treatment of DED, a number of limitations remain. Firstly, the causes of DED are varied, including topical or systemic disease. Secondly, the severity of DED in the patients included in the seven studies was inconsistent. Three $[26,29,32]$ of the studies included 52 subjects with severe DED, one study [27] included 96 subjects with moderate to severe DED, and the others did not identify 
the severity of DED $[24,25,33]$. Thirdly, there were differences in the dosage, frequency, and treatment course in the seven studies, which potentially affected prognosis to some extent.

As for the concentration of serum, five studies used $20 \%$, one study used $40 \%$, and another used $50 \%$. Consensus for the optimal concentration has never been established. In consideration of this review focus on comparing the efficiency of AT and AS, and the concentration of effectiveness ingredients may differ among individuals, we included studies using AS for the intervention measure. All included studies used AT as a control. Concretely, three of the seven included trials used preservative-free AT as a control. One trial used Systane, and another used Refresh. The other two studies did not clearly state what kind of AT was used.

In conclusion, the meta-analyses conclude that AS treatment may provide some benefit to DED patients on OSDI, TBUT, and rose bengal staining score compared with AT treatment. The different classifications of DED and the inconsistent concentration of AS used in these studies were the main limitations. Thus, large sample RCTs with standardized protocols are needed to further evaluate the exact role of AS in the management of DED.

\section{Statement of Ethics}

All analyses were based on published studies, and thus, no ethical approval and informed consent are required. Nonetheless, the study adhered fully to the Declaration of Helsinki.

\section{Disclosure Statement}

No conflicting relationship exists for any author.

\section{Funding Sources}

Our research was supported by National Nature Science Foundation of China (grant No. 81970765) and Beijing Science and Technology Project (Z181100001918031), China.

\section{Author Contributions}

L.W. and K.C. extracted data from published studies, analyzed the results, and drafted the manuscript. They contributed equally to the study. Z.W. provided oversight for the extraction and analysis. C.B., A.L., and Q.L. ensured that the analysis was conducted appropriately and identified the accuracy of results and discussion presented in the manuscript.

\section{References}

1 Craig JP, Nichols KK, Akpek EK, Caffery B, Dua HS, Joo C-K, et al. TFOS DEWS II Definition and Classification Report. Ocul Surf 2017;15(3):276-83.

2 Sickenberger W. Eins, Zwei, Drei - A German's perspective on dry eye numbers in the world. Cont Lens Anterior Eye. 2017 Feb;40(1):1-2.

3 Tan LL, Morgan P, Cai ZQ, Straughan RA. Prevalence of and risk factors for symptomatic dry eye disease in Singapore. Clin Exp Optom. 2015 Jan;98(1):45-53.

4 Song $\mathrm{P}$, Xia W, Wang $\mathrm{M}$, Chang X, Wang J, Jin S, et al. Variations of dry eye disease prevalence by age, sex and geographic characteristics in China: a systematic review and metaanalysis. J Glob Health. 2018 Dec;8(2):020503.

5 Stapleton F, Alves M, Bunya VY, Jalbert I, Lekhanont K, Malet F, et al. TFOS DEWS II Epidemiology Report. Ocul Surf 2017;15(3): 334-65.

6 Jones L, Downie LE, Korb D, Benitez-DelCastillo JM, Dana R, Deng SX, et al. TFOS DEWS II Management and Therapy Report. Ocul Surf. 2017 Jul;15(3):575-628.

7 Drew VJ, Tseng CL, Seghatchian J, BurnoufT. Reflections on Dry Eye Syndrome Treatment: Therapeutic Role of Blood Products. Front Med (Lausanne). 2018 Feb;5:33.

8 López-García JS, García-Lozano I, Rivas L, Giménez C, Acera A, Suárez-Cortés T. Effects of Autologous Serum Eye Drops on Conjunctival Expression of MUC5AC in Patients With Ocular Surface Disorders. Cornea. 2016 Mar;35(3):336-41.

9 Esquenazi S, He J, Bazan HE, Bazan NG. Use of autologous serum in corneal epithelial defects post-lamellar surgery. Cornea. 2005 Nov;24(8):992-7.

10 Poon AC, Geerling G, Dart JK, Fraenkel GE, Daniels JT. Autologous serum eyedrops for dry eyes and epithelial defects: clinical and in vitro toxicity studies. Br J Ophthalmol. 2001 Oct;85(10):1188-97.

11 Rocha EM, Pelegrino FS, de Paiva CS, Vigorito AC, de Souza CA. GVHD dry eyes treated with autologous serum tears. Bone Marrow Transplant. 2000 May;25(10):1101-3.

12 Ogawa Y, Okamoto S, Mori T, Yamada M, Mashima Y, Watanabe R, et al. Autologous serum eye drops for the treatment of severe dry eye in patients with chronic graft-versushost disease. Bone Marrow Transplant. 2003 Apr;31(7):579-83.

13 Ralph RA, Doane MG, Dohlman CH. Clinical experience with a mobile ocular perfusion pump. Arch Ophthalmol. 1975 Oct;93(10): 1039-43.

14 Noble BA, Loh RS, MacLennan S, Pesudovs K, Reynolds A, Bridges LR, et al. Comparison of autologous serum eye drops with convention- al therapy in a randomised controlled crossover trial for ocular surface disease. Br J Ophthalmol. 2004 May;88(5):647-52.

15 Tananuvat N, Daniell M, Sullivan LJ, Yi Q, McKelvie P, McCarty DJ, et al. Controlled study of the use of autologous serum in dry eye patients. Cornea. 2001 Nov;20(8):8026.

16 Higgins JPT, Green S (eds). Cochrane Handbook for Systematic Reviews of Interventions Version 5.1.0 [updated March 2011]. The Cochrane Collaboration, 2011. Available from www.cochrane-handbook.org.

17 Borenstein M, Hedges LV, Higgins JP, Rothstein HR. A basic introduction to fixed-effect and random-effects models for meta-analysis. Res Synth Methods. 2010 Apr;1(2):97111.

18 Egger M, Davey Smith G, Schneider M, Minder C. Bias in meta-analysis detected by a simple, graphical test. BMJ. 1997 Sep;315(7109):629-34.

19 Pan Q, Angelina A, Marrone M, Stark WJ, Akpek EK. Autologous serum eye drops for dry eye. Cochrane Database Syst Rev. 2017 Feb;2:CD009327.

20 Wan KH, Chen LJ, Young AL. Efficacy and Safety of Topical $0.05 \%$ Cyclosporine Eye Drops in the Treatment of Dry Eye Syndrome: A Systematic Review and Meta-analysis. Ocul Surf. 2015 Jul;13(3):213-25. 
21 Giannaccare G, Pellegrini M, Sebastiani S, Bernabei F, Roda M, Taroni L, et al. Efficacy of Omega-3 Fatty Acid Supplementation for Treatment of Dry Eye Disease: A Meta-Analysis of Randomized Clinical Trials. Cornea. 2019 May;38(5):565-73.

22 Song JK, Lee K, Park HY, Hyon JY, Oh SW, Bae WK, et al. Efficacy of Carboxymethylcellulose and Hyaluronate in Dry Eye Disease: A Systematic Review and Meta-Analysis. Korean J Fam Med. 2017 Jan;38(1):2-7.

23 Ang BC, Sng JJ, Wang PX, Htoon HM, Tong LH. Sodium Hyaluronate in the Treatment of Dry Eye Syndrome: A Systematic Review and Meta-Analysis. Sci Rep. 2017 Aug;7(1):9013.

24 Semeraro F, Forbice E, Nascimbeni G, Taglietti M, Romano V, Guerra G, et al. Effect of Autologous Serum Eye Drops in Patients with Sjögren Syndrome-related Dry Eye: Clinical and In Vivo Confocal Microscopy Evaluation of the Ocular Surface. In Vivo. 2016;30(6): $931-8$.
25 Yılmaz U, Küçük E, Koç Ç, Gökler E. Comparison of Autologous Serum Versus Preservative Free Artificial Tear in Patients with Dry Eyes Due to Systemic Isotretinoin Therapy. Curr Eye Res. 2017 Jun;42(6):827-31.

26 Kojima T, Ishida R, Dogru M, Goto E, Matsumoto Y, Kaido M, et al. The effect of autologous serum eyedrops in the treatment of severe dry eye disease: a prospective randomized case-control study. Am J Ophthalmol. $2005 \mathrm{Feb} ; 139(2): 242-6$.

27 Mukhopadhyay S, Sen S, Datta H. Comparative role of $20 \%$ cord blood serum and $20 \%$ autologous serum in dry eye associated with Hansen's disease: a tear proteomic study. Br J Ophthalmol. 2015 Jan;99(1):108-12.

28 Tsubota K, Xu KP, Fujihara T, Katagiri S, Takeuchi T. Decreased reflex tearing is associated with lymphocytic infiltration in lacrimal glands. J Rheumatol. 1996 Feb;23(2):313-20.
29 Urzua CA, Vasquez DH, Huidobro A, Hernandez $\mathrm{H}$, Alfaro J. Randomized doubleblind clinical trial of autologous serum versus artificial tears in dry eye syndrome. Curr Eye Res. 2012 Aug;37(8):684-8.

30 Valim V, Trevisani VF, Pasoto SG, Serrano EV, Ribeiro SL, Fidelix TS, et al. Recommendations for the treatment of Sjögren's syndrome. Rev Bras Reumatol. 2015 Sep-Oct; 55(5):446-57.

31 Doughty MJ. Rose bengal staining as an assessment of ocular surface damage and recovery in dry eye disease-a review. Cont Lens Anterior Eye. 2013 Dec;36(6):272-80.

32 Celebi AR, Ulusoy C, Mirza GE. The efficacy of autologous serum eye drops for severe dry eye syndrome: a randomized double-blind crossover study. Graefes Arch Clin Exp Ophthalmol. 2014 Apr;252(4):619-26.

33 Noda-Tsuruya T, Asano-Kato N, Toda I, Tsubota K: Autologous serum eye drops for dry eye after LASIK. J Refract Surg. 2006 JanFeb;22(1):61-6. 